\title{
Implications of Education and Prehabilitation on the Geriatric Surgical Patient
}

Hufford, Theadore $\mathrm{MD}^{1}$; Rubin, Jonathan $\mathrm{MD}^{1}$; Al-Qudah, Ghaith ${ }^{1}$; Prendergast, Michael MD2

${ }^{1}$ University of Illinois, Metropolitan Group Hospitals General Surgery Residency, Advocate Illinois Masonic Hospital, Chicago, IL, USA

${ }^{2}$ Presence Saint Francis Hospital, Department of Surgery, Evanston, IL, USA

Mailing Address: 836 W. Wellington, Room 4807 Chicago, IL 60657

Phone number: 937-681-0777

Email: tjhufford80@gmail.com 


\section{INTRODUCTION}

Recently there has been a significant increase in age in the United States. It is necessary to better understand the physiological and surgical needs of these patients in order to optimize outcomes. The vast majority of procedures performed in adult patients are lowrisk operations, such as laparoscopic cholecystectomy. Our aim is to investigate the outcomes, including length of stay, morbidity, mortality, re-admission and discharge disposition of the elderly population $(>80)$ undergoing low-risk operations in our tertiary community hospital.

\section{METHODS}

A retrospective chart review was done at a tertiary community hospital. Inpatient and outpatient records were evaluated. The time frame utilized was 2011-2015. Patients were excluded only on the basis of their age $($ age $<80)$ at the time of operation. Data collected was used to compare the overall general health of this population, morbidity and mortality associated with the operation, hospital length of stay, and discharge disposition. Approval to conduct this retrospective chart review was obtained by the institution's Institutional Review Board.

\section{RESULTS}

There were a total of 30 patients who underwent laparoscopic cholecystectomy from 2011 to 2015. 21 patients $(70 \%)$ were female and $9(30 \%)$ were male. No patients were converted to an open procedure. The average age was 86.4 years and average ASA classification prior to surgery was 2.88 . Higher ASA class, specifically those that were class III/IV were more likely to have an increased length of stay that was statistically significant. Overall age greater than 80 was an independent risk factor for transfer to a higher level of care upon discharge (SNF, LTAC, etc.), a surrogate marker for physical decompensation following surgery.

\section{CONCLUSIONS}

Routine surgery, such as the laparoscopic cholecystectomy, effects the elderly population in a more substantial way, and early recognition coupled with increased education for physicians regarding geriatric patients can help to reduce length of stay, morbidity, and overall physical and mental deconditioning. 


\section{INTRODUCTION}

It has been well established that in recent years, there has been and will continue to be a significant increase in age among the United States population. Specifically, the elderly population (defined as an age greater than 65) has been growing at an exponential rate. From 2000 to 2010 , the elderly population increased at a rate of $15.1 \%$, while the total United States population lagged behind with an overall growth rate of $9.1 \%$. By 2030 it 71 is estimated that 70 million people will be over 65 years of age, which will account for approximately $20 \%$ of the overall U.S. population ${ }^{1,6}$. The elderly population will increase by $100 \%$ to almost 90 million by the year $2050 \%$. With this dramatic shift in the demographic make-up of the American population, it is the responsibility of the medical community to better understand the physiological and surgical needs of these patients in order to better optimize outcomes, thus providing better overall care.

When addressing this patient population, it is crucial to understand the physiologic changes that occur with age. Age related physiological changes are becoming more and more understood with this aging population. Significant work has been done to show differences in this elderly population in areas like cardiac output, thermoregulation, baroreceptor response, vital capacity, airway clearance, partial pressure of oxygen, and muscle mass

The American College of Surgeons has published an updated statement and guidelines in 2016 regarding nine assessment categories corresponding to cognitive/behavioral disorders, cardiac evaluation, pulmonary evaluation, functional/performance status, frailty, nutritional status, medication management, patient counseling, and preoperative testing. These guidelines will help surgeons better understand the risks of the geriatric population in order to reduce morbidity and mortality in this group; however, the primary focus thus far in geriatric surgery research has been on higher risk procedures. The vast majority of procedures performed for elderly patients are low-risk operations, such as the

laparoscopic cholecystectomy 4,5 . Our aim is to investigate the differences in outcomes, including length of stay, morbidity and mortality of this population compared with accepted norms published in the literature in the younger population.

\section{METHODS}

A retrospective chart review was done at a tertiary community hospital. Inpatient and outpatient records were evaluated. The time frame utilized was 2011-2015. Patients were excluded only on the basis of their age $($ age $<80)$ at the time of operation. Data collected was used to compare the overall general health of this population, morbidity and mortality associated with the operation, hospital length of stay, and discharge disposition. Approval to conduct this retrospective chart review was obtained by the institution's Institutional Review Board. 


\section{RESULTS}

There were a total of 30 patients who underwent laparoscopic cholecystectomy from 2011 to 2015.21 patients $(70 \%)$ were female and $9(30 \%)$ were male. No patients were converted to an open procedure. The average age was 86.4 years and average ASA classification prior to surgery was 2.88 . Overall demographic data is illustrated in table 1 . Higher ASA class, as shown in table 2, specifically those that were class III/IV were more likely to have an increased inpatient hospital length of stay that was statistically significant. Overall age $>80$ was an independent risk factor for transfer to a higher level of care upon discharge, which can be used as a surrogate marker for physical decompensation following surgery (Table 3 ).

\section{DISCUSSION}

The American College of Surgeons updated list of assessment tools published in 2016 provides a guide to assist the surgical and medical team in the care of the geriatric population, but additional research can and should focus on the area of pre-operative assessment and post-operative discharge planning. Routine surgery, such as the laparoscopic cholecystectomy, has a more dramatic effect in a substantial way. Early recognition of known age related physiological changes coupled with increased education for clinicians regarding geriatric patients could help to reduce length of stay, morbidity, and overall physical and mental deconditioning.

Some age related physiological changes that are important to note include decreased muscle mass and increased adipose tissue. In the post-operative period this is clinically significant when managing the geriatric patient due to a decreased in mobility, impairment of cough reflex, and the increased in overall risk for deep vein thrombosis. Addressing these risks factors by providing early nutrition and limiting mobility reducing factors (i.e., drains, restraints, catheters) should be considered ${ }^{2}$. Early initiation of physical and occupational therapy can help to limit the mobility concerns while early initiation of DVT chemoprophylaxis can also ameliorate VTE risk.

The cardiovascular geriatric perspective represents its own unique set of challenges. It has been consistently shown that they have a decrease in cardiac output, thermoregulation, and baroreceptor response and are predisposed to dysrhythmias, hypothermia, and hypovolemia. Cardiac complications are the leading cause of perioperative mortality and morbidity among all age groups, and the geriatric population is at even higher risk. Vigorous, yet judicious and conscientious, fluid resuscitation and the use of non-vasoconstricting inotropes are important management practices to use in the correct setting ${ }^{2}$.

Decreased vital capacity, impaired airway clearance, and limited partial pressure of oxygen are known physiologic respiratory changes that occur in the elderly. In the postoperative period, this predisposes the geriatric surgical patient to an increased risk of atelectasis, aspiration, and hypoxemia. These known limitations further demonstrate the 
need to promote early ambulation by reducing factors that limit mobility, and also the importance of providing supplemental oxygen in a more routine fashion ${ }^{2}$.

Geriatric patients, as suggested by our data and as would make logical sense, have a higher average ASA class prior to operation. As was shown here, the higher preoperative ASA class the more lengthy hospital stay. Earlier recognition of this by all members of the care team on initial presentation and prior to operation, when feasible, could help the physician to prepare for likely discharge delays in a more pro-active way. Increased education for surgeons, clinicians, and ancillary staff could make a significant impact on estimated increased hospital length of stay, beginning with the initial preparations for discharge disposition needs upon admission.

It is a well-established fact in the medical literature that complicated gallstone disease is more prevalent in the elderly than the younger patient population. Further-more, performing laparoscopic cholecystectomy on elderly patients has resulted in increased postoperative complications such as increased morbidity, biliary tract disease, and longer hospital stays ${ }^{7}$. These severe postoperative outcomes have been attributed to the lack of muscle strength and poor physical condition associated with the older patient population . Though studies have found that laparoscopic cholecystectomy may cause less post- operative complications in general, it is still linked to longer operation time, pro-longed hospital stay, and higher morbidity in geriatric patients compared to the younger ones .

It should also be noted that a large majority of patients did not return to their previous living environment (i.e. those at home were more likely to go to an ECF or rehabilitation facility upon discharge). This process, though limited by the Medicare system and some requirements needed to qualify for these specific disposition plans, can also be limited by the patient, family, hospital support staff and social workers preparing for these things upon the readiness for discharge rather than using foresight, as well as the data presented here, to ready the patient for an alternative disposition.

Pre-operative assessment and proactive discharge planning that begins upon admission when the plan of care involves routine surgery in the geriatric patient (greater than 80 years of age), such as the laparoscopic cholecystectomy, may play a significant role in this early recognition coupled with increased education for clinicians can help to reduce length of stay, morbidity, and overall physical and mental deconditioning.

Despite the increasing number of elderly patients undergoing laparoscopic surgery along with the associated co-morbidity factors, there is still a lack of research studies investigating risks older patients endure after undergoing such procedures . Therefore, defining patient segments (such as geriatric patients) who are specifically at risk of postoperative complications and standardizing the outcome is of extreme importance to improve postoperative results. 
In fact, investigating the postoperative outcomes in the geriatric population will have dramatic implications on prehabilitation where the preoperative condition of a patient is enhanced as a means to improve his/her postoperative outcome. This can be a valid option in patients going for elective laparoscopic cholecystectomy. The inclusion of prehabilitation in the geriatric population and the initiation of these tools within the formal geriatric education that surgical residents receive could greatly benefit the overall patient outcome and decrease some current intangible burden on the overall healthcare system.

\section{CONCLUSIONS}

Routine surgery, such as the laparoscopic cholecystectomy, effects the elderly population in a more substantial way, and early recognition coupled with increased education for physicians regarding geriatric patients can help to reduce length of stay, morbidity, and overall physical and mental deconditioning. Future work on this will be to explore the possibility of implementing systems-based practice screening tools that can be used to be identify those that might benefit from prehabilitation exercises and the inclusion of prehabilitation within the geriatric education already implemented for surgical residents has the potential to improve outcomes for these patients and the utility of these tools can easily be assessed in future research in this area.

Acknowledgements: We would like to thank Dr. Matthew Hyser and Dr. Charles Gruner for their input the process as well for their participation in the direct care of these patients and this particular patient population.

\section{REFERENCES}

1. Werner C. The Older Population: 2010. Washington, DC: U.S. Census Bureau; 2011.

2. Watters JM: Surgery in the elderly. Can K Surg 45: 106, 2002.

3. Mohanty, S., Rosenthal, R.A., Russell, M.M., Neuman, M. D., Ko, C.Y., \& Esnaola, N.F. (2016). Optimal Perioperative Management of the Geriatric Patient: A Best Practices Guideline from the American College of Surgeons, 22(5), 918-922.

4. Kirshtein, B., Perry, Z.H., Mizrahi, S., \& Lantsberg, L. (2009). Value of the Laparoscopic Appendectomy in the Elderly Patient. World J Surg World Journal of Surgery, 33(5), 918-922.

5. Agrusa, A., Romano, G., Franzzetta, G., Chianetta, D., Sorce, V., Buono, G.D., \& Gulotta, G. (2014) Role and outcomes of laparoscopic cholecystectomy in the elder. International Journal of Surgery, 12.

6. Ortman, J.M., Velkoff, V.A., \& Hogan, H. (2014). An Aging Nation: The Older Population in the United States. Population Estimates and Progjections, Current Population Report P25-1140. U.S. Department of Commerce, Economics and Statistics Administration, U.S. Census Bureau. 
7. Bruns, E.R., van den Huevel, B., Buskens, C.J., van Duijvendijk, P., Festen S., Wassenaar, E.B., van der Zaag, E. S., Bemelman, W. A., \& van Munster, B.C. (2016). The Effects of Physical Prehabilitation in Elderly Patients Undergoing Col-orectal Surgery: A Systematic Review. Colorectal Dis., 18(8), O267-77.

8. Caglia, P., Costa, S., Tracia, A., Veroux, M., Luca, S., Zappulla, E., Russo, V., Lu- cifora, B., Brozi, L., Patate, G., Trovato, S., \& Amedeo, C. (2012). Can Laparoscopic Cholecystectomy Be Safety Performed in the Elderly?. Ann Ital Chir, 83(1), 21-4.

9. Qasaimeh, G.R., \& Banihani, M.N. (2012). Laparoscopic Cholecystectomy in the Elderly and Young: A Comparative Study. Hepatogastroenterology, 59(113), 225.

\section{LEGENDS}

\begin{tabular}{|c|c|c|c|}
\hline \multirow{nyy}{*}{ Sex } & Male & Female & Total \\
\hline Age & 9 & 21 & 30 \\
\cline { 2 - 4 } & $30.00 \%$ & $70.00 \%$ & $100.00 \%$ \\
\hline Lap. Cholecystectomy & 87.22 & 86.09 & 86.41 \\
\hline ASA Score & $88.89 \%$ & $95.83 \%$ & $93.75 \%$ \\
\hline Previous Abd Surgery & 2.89 & 2.87 & 2.88 \\
\hline Readmission & $22.56 \%$ & $65.22 \%$ & $2.50 \%$ \\
\hline
\end{tabular}

Table 1: Overall Demographics

\begin{tabular}{|c|c|c|c|}
\cline { 2 - 4 } & Average Age & Length of Stay (Days) & p value \\
\hline ASA 1 & 86.0 & 1.0 & 1 \\
\hline ASA 2 & 84.4 & 5.0 & 0.0469 \\
\hline ASA 3 & 86.4 & 8.0 & 0.0000 \\
\hline ASA 4 & 89.7 & 12.3 & 0.0028 \\
\hline
\end{tabular}

Table 2: ASA Classes for each patient

\begin{tabular}{|c|c|c|c|}
\hline Age & Length of Stay & Disposition Not Home & p value \\
\hline $80-84$ & 7.36 & $27.27 \%$ & 0.0264 \\
\hline $85-89$ & 8.14 & $50.00 \%$ & 0.0009 \\
\hline $90+$ & 7.57 & $57.14 \%$ & 0.0023 \\
\hline Overall & 7.75 & $43.75 \%$ & 0.0000 \\
\hline
\end{tabular}

Table 3: Average LOS and Percent of Patients being discharged to higher acuity status 